Research Article; Received: May 25, 2021; Accepted: June 21, 2021

\title{
ON BIVARIATE EXTENSION OF THE UNIVARIATE TRANSMUTED DISTRIBUTION FAMILY
}

\author{
Mehmet YILMAZ and Hüseyin ÜNÖZKAN \\ Statistics Department, Ankara University, Ankara, TURKEY
}

\begin{abstract}
The aim of this study is to examine the bivariate transmuted distributions in the literature and to propose alternative distribution. The method is based on mixing distributions of pairs of order statistics of a sample of size two. Some of proposed distributions allow both negative and positive Pearson correlations with admissible range between pairs of random variates. The results of the study gain importance in terms of eliminating or completing the missing aspects of the bivariate transmuted distributions existing in the literature.
\end{abstract}

\section{INTRODUCTION}

Quadratic Rank Transmutation Mapping (QRTM) method proposed by Shaw and Buckley (2009) 20, which is one of the most popular methods of constructing univariate distribution. In multivariate data modelling, instead of investigating the independence between random variables, sometimes one can desire to construct a multivariate distribution of highly correlated random variables. For this purpose, it is important to extend the QRTM technique to two-dimensional distributions both in terms of providing flexibility and making alternative distribution suggestions to the previous works.

Some authors have worked with the bivariate (or multivariate) extension of the univariate transmuted distributions. The bivariate and multivariate extensions of the univariate-transmuted family are first introduced by Bourguignon et al. (2016) 7. They obtained continuous bivariate and multivariate distributions having transmuted univariate marginals. Merovci et al. (2016) 11] introduced the bivariate version of univariate generalized transmuted G distribution. Alizadeh et

2020 Mathematics Subject Classification. 60E05 62H05 62H10.

Keywords. Bivariate extension, transmuted distribution, dependence, bivariate distribution, Spearman's Rho.

■mehmetyilmaz@ankara.edu.tr-Corresponding author; hunozkan@gmail.com

(D) 0000-0002-9762-6688; 0000-0001-9659-287X. 
al. (2017) [1] introduced the bivariate version of univariate generalized transmuted distribution. Unlike the works of Bourguignon et al. (2016) 7] and Merovci et al. (2016) 11], Alizadeh et al. (2017) [1] considered the base distribution as an exponentiated distribution. Merovci et al. (2017) [12 proposed the bivariate extension of the univariate Exponentiated-Transmuted G (ET-G) distribution family. Rezaei et al. (2017) [16 introduced a bivariate version of the generalized exponentiated distribution (GEC-G) by using failure probabilities of nested series and parallel systems. Bakouch et al. (2017) 4] proposed a bivariate version of the transmuted general (T-G) family of distributions. Although Bakouch et al. (2017) [4] use of similar techniques, unlike other authors' works, they proposed the Bivariate T-G distribution by considering different baseline distribution. Unlike the works mentioned above, Sarabia et al. 18 suggests a bivariate distribution with transmuted conditionals.

According to Table 1 of Shaw and Buckley (2009) 20], the essence of the univariate distributions derived from the QRTM is based on minimum and maximum order statistics, therefore this technique may not be easy to apply for bivariate or multivariate distributions. Since a bivariate distribution is constructed over the quadrants, the complement of the bivariate distribution is different from the univariate case.

Some relevant references about construction bivariate and multivariate distributions based on the pairs of order statistics are in [3], [8], [14 and 13]. According to work of Dolati and Úbeda-Flores (2009) [8], a bivariate distribution family was introduced by assuming negative dependence [6] and [21].

Since we inspire by the works of [8] and [13] in the present study, let's give these studies in more detail as follows: Dolati and Úbeda-Flores (2009) 8 introduced two transformations, based on the choice of pairs of order statistics of the marginal distributions. [13] defined that the bivariate distribution having the transmuted marginals, both by examining the univariate QRTM technique and inspired by [8].

In this study, we first examined the bivariate extensions that exist in the literature, and secondly, we discussed the new distribution proposals in the light of the studies of 8 and $[13$. The findings obtained as a result of the study show that the proposed distributions can be used as an alternative to the extensions in the literature without any restrictions on the base distribution.

Accordingly, the study is organized as follows: The material and method section will be presented in four parts, these are as follows: Brief introduction of univariate QRTM given by Shaw and Buckley (2009) [20]. The extension of the QRTM technique to the bivariate case proposed by [7] will be considered, and we will discuss by Theorem 1 under which conditions this extension will be a bivariate distribution. Based on the studies of [8] and [13, the introduction of the technique to be used for new distribution proposals will be detailed. 
In section 3, new distribution suggestions will be made, and Spearman's rank correlation coefficient will be calculated based on a specially selected distribution as base distribution.

The last part of the study includes the comparison of the proposed distributions with the studies available in the literature, a discussion of the advantages and disadvantages.

\section{Material and Method}

The quadratic rank transmutation mapping (QRTM) proposed by Shaw and Buckley (2009) $[20$ is given as $u \longrightarrow u+\lambda u(1-u)$, where $u \in[0,1]$ and $\lambda \in[-1,1]$. We come up with an idea inspired by Shaw and Buckley (2009) 20] as follows:

Let $X_{1}$ and $X_{2}$ be two independent and identically distributed random variables. Then once recall the distributions of order statistics associated with sample size of 2:

$$
\begin{gathered}
\operatorname{Pr}\left(X_{1: 2} \leq x\right)=1-\operatorname{Pr}\left(X_{1: 2}>x\right)=1-(1-F(x))^{2}=2 F(x)-F^{2}(x), \\
\operatorname{Pr}\left(X_{2: 2} \leq x\right)=F(x)^{2} .
\end{gathered}
$$

Now, a new random variable $T$ is defined by mixing the above order statistics as follows:

$$
T= \begin{cases}X_{1: 2}, & \text { with probability } \pi_{1} \\ X_{2: 2}, & \text { with probability } \pi_{2},\end{cases}
$$

where $\pi_{1}+\pi_{2}=1$. Then the distribution of $T$ is as follows:

$$
\begin{aligned}
\operatorname{Pr}(T \leq t) & =\pi_{1}\left(2 F(t)-F^{2}(t)\right)+\pi_{2}\left(F^{2}(t)\right) \\
& =\left(2 \pi_{1}\right) F(t)+\left(\pi_{2}-\pi_{1}\right) F^{2}(t) .
\end{aligned}
$$

By letting $\pi_{2}=1-\pi_{1}$ then we have

$$
\operatorname{Pr}(T \leq t)=2 \pi_{1} F(t)+\left(1-2 \pi_{1}\right) F^{2}(t) .
$$

Since $\pi_{1} \in[0,1]$, appropriate parametrization for $\pi_{1}$ can be taken into account as $2 \pi_{1}=1+\lambda$. New parameter is in the interval $[-1,1]$. Accordingly, latter probability is as follows:

$$
\begin{aligned}
\operatorname{Pr}(T \leq t) & =(1+\lambda) F(t)-\lambda F^{2}(t) \\
& =F(t)+\lambda F(t)(1-F(t)),
\end{aligned}
$$

with $\lambda \in[-1,1]$. As can be seen immediately, if $F(t)=u$ is taken into account, the above expression is the quadratic rank transmutation proposed in $[20$. Here, $F(t)$ is named as "base distribution" and $\operatorname{Pr}(T \leq t)=G(t)$ is named as "transmuted distribution". 
Based on this, bivariate extension of quadratic transmuted distribution proposal of [7] draws our attention in particular. They proposed quadratic rank transmuted bivariate distribution as

$$
H(x, y)=(1+\lambda) F(x, y)-\lambda F(x, y)^{2},
$$

where $\lambda \in[-1,1]$. Marginals of this model are univariate transmuted distributions respectively as $(1+\lambda) F_{x}(x)-\lambda F_{x}(x)^{2}$ and $(1+\lambda) F_{y}(y)-\lambda F_{y}(y)^{2}$.

Furthermore, just like as in the univariate case, a bivariate distribution should be obtained with the eq. (1) for the extreme values of $\lambda$. Such that for $\lambda=-1$, eq. (1) reduces to give $F(x, y)^{2}$ which is a cumulative distribution function (cdf), and for $\lambda=1$, eq. (1) gives $2 F(x, y)-F(x, y)^{2}$ which is not a cdf.

Furthermore, $2 F(x, y)-F(x, y)^{2}$ indicates the probability that is

$$
\operatorname{Pr}\left(\left\{X_{1} \leq x, Y_{1} \leq y\right\} \cup\left\{X_{2} \leq x, Y_{2} \leq y\right\}\right),
$$

where $\left(X_{1}, Y_{1}\right)$ and $\left(X_{2}, Y_{2}\right)$ are independent and identically distributed copulas from $F$. Therefore, there are some issues to overcome for the case of positive values of the transmutation parameter $\lambda$. To overcome these issues, we have the following theorem.

Theorem 1. Let $F(x, y)$ be a continuous distribution function and $H(x, y)$ be a differentiable on $\Re^{2}$ where $h(x, y)$ denotes $\frac{\partial^{2} H(x, y)}{\partial x \partial y}$. Then $H(x, y)=(1+\lambda) F(x, y)-$ $\lambda F(x, y)^{2}$ is a continuous distribution function, if the following conditions hold:

(i) $-1 \leq \lambda \leq 0$,

(ii) $0<\lambda \leq \frac{1}{3}$ and $F(x, y)$ belongs to Positively Dependent Class.

Proof. Multivariate distribution function must satisfy $((\mathrm{P} 1)-(\mathrm{P} 3))$ properties (see, Barlow and Proschan, 1975, Chapter 5 [5]). The properties (P1) and (P2) are obviously hold. We prove only (P3).

(i)

(P3) $\frac{\partial^{2} H(x, y)}{\partial x \partial y} \geq 0$. For the simplicity, let $f_{x y}=f(x, y), F_{x y}=F(x, y)$, and $h_{x y}=\frac{\partial^{2} H(x, y)}{\partial x \partial y}$. Then

$$
h_{x y}=f_{x y}\left[1+\lambda-2 \lambda F_{x y}\right]-2 \lambda \frac{\partial F_{x y}}{\partial x} \frac{\partial F_{x y}}{\partial y}
$$

where $\frac{\partial F_{x y}}{\partial x}$ and $\frac{\partial F_{x y}}{\partial y}$ are respectively the probabilities of $\operatorname{Pr}(Y \leq y, X=x)$ and $\operatorname{Pr}(X \leq x, Y=y)$. Obviously, from the eq.[2], for $\lambda \in[-1,0], h_{x y} \geq 0$.

(ii)

(P3) Under the assumption of the positive dependence of $F$, according to [15 and 2 , by noting that positive dependence implies $f_{x y} F_{x y} \geq \frac{\partial F_{x y}}{\partial x} \frac{\partial F_{x y}}{\partial y}$. Hence, by the eq.(2), we have 


$$
\begin{aligned}
h_{x y} & \geq f_{x y}\left[1+\lambda-4 \lambda F_{x y}\right] \\
& \geq f_{x y}[1-3 \lambda] .
\end{aligned}
$$

By considering $\lambda \leq 1 / 3$, the latter expression in square brackets is non negative. This completes the proof.

The transition from univariate case to bivariate or multivariate cases is not so easy. While in univariate case the real line is the complement of $\operatorname{Pr}(X \leq x)$, at least in the bivariate case the complement of $\operatorname{Pr}(X \leq x, Y \leq y)$ is on the quadrants.

In response to the above discussions, we consider the work of Dolati and ÚbedaFlores (2009) 8. They introduced a method for constructing bivariate distribution by using order statistics.

Let $\left(X_{1}, Y_{1}\right)$ and $\left(X_{2}, Y_{2}\right)$ be two independent random vectors with common distribution function $F(x, y)$. Note that, $F(x, y)$ belongs to the distribution family $\mathcal{F}\left(F_{x}, F_{y}\right)$ where $F_{x}$ and $F_{y}$ denote respectively marginals of $X$ and $Y$. Let $X_{(1)}$, $X_{(2)}$ and $Y_{(1)}, Y_{(2)}$ be their corresponding order statistics. According to 8 , consider the four probabilities as follows:

$$
\begin{gathered}
\operatorname{Pr}\left(X_{(1)} \leq x, Y_{(2)} \leq y\right)=F_{x y}\left(2 F_{y}-F_{x y}\right), \\
\operatorname{Pr}\left(X_{(2)} \leq x, Y_{(1)} \leq y\right)=F_{x y}\left(2 F_{x}-F_{x y}\right), \\
\operatorname{Pr}\left(X_{(1)} \leq x, Y_{(1)} \leq y\right)=2 F_{x} F_{y}+2 F_{x y} \bar{F}_{x y}-F_{x y}^{2}
\end{gathered}
$$

and

$$
\operatorname{Pr}\left(X_{(2)} \leq x, Y_{(2)} \leq y\right)=F_{x y}^{2},
$$

where $\bar{F}_{x y}$ denotes survival function of $(X, Y)$ i.e., $\operatorname{Pr}(X>x, Y>y)$. Dolati and Úbeda-Flores (2009) 8 proposed two new distributions. First is a mixture of (3) and (4) with mixing probability $\frac{1}{2}$. In other words,

$$
\left(Z_{1}, Z_{2}\right)=\left\{\begin{array}{l}
\left(X_{(1)}, Y_{(2)}\right), \text { with probability } \frac{1}{2} \\
\left(X_{(2)}, Y_{(1)}\right), \text { with probability } \frac{1}{2} .
\end{array}\right.
$$

Then the distribution of $\left(Z_{1}, Z_{2}\right)$ is given by

$$
H_{1}(x, y)=F_{x y}\left[1-\bar{F}_{x y}\right] .
$$

Second is a mixture of (5) and (6) with mixing probability $\frac{1}{2}$. In other words, If we consider the random vector

$$
\left(T_{1}, T_{2}\right)=\left\{\begin{array}{l}
\left(X_{(1)}, Y_{(1)}\right), \text { with probability } \frac{1}{2} \\
\left(X_{(2)}, Y_{(2)}\right), \text { with probability } \frac{1}{2},
\end{array}\right.
$$

the distribution function of $\left(T_{1}, T_{2}\right)$ is given by 


$$
H_{2}(x, y)=F_{x} F_{y}+F_{x y} \bar{F}_{x y} .
$$

Note that, $H_{1}$ and $H_{2}$ both belong to $\mathcal{F}\left(F_{x}, F_{y}\right)$. Accordingly, a transmuted bivariate distribution was introduced by considering the eq. (5) and eq. (6) in 13 as follows: If we consider the random vector

$$
\left(T_{1}^{*}, T_{2}^{*}\right)=\left\{\begin{array}{l}
\left(X_{(1)}, Y_{(1)}\right), \text { with probability } \frac{1+\lambda}{2} \\
\left(X_{(2)}, Y_{(2)}\right), \text { with probability } \frac{1-\lambda}{2},
\end{array}\right.
$$

for $\lambda \in[-1,1]$, the distribution function of $\left(T_{1}^{*}, T_{2}^{*}\right)$ is given by

$$
H_{3}(x, y)=(1+\lambda)\left[F_{x} F_{y}+F_{x y} \bar{F}_{x y}\right]-\lambda F_{x y}^{2} .
$$

Note that, $H_{3}$ does not belong to $\mathcal{F}\left(F_{x}, F_{y}\right)$. Marginals are represented by univariate transmuted distribution as in (1). With descriptions so far, it can easily be said that the genesis of the eq. (9) is one of the bivariate extension of QRTM.

Next section, we try to propose three alternative distributions. The first and second are proposed in the light of the works of [8] and [13. The third proposal is based on QRTM technique of [20].

\section{The Research Findings and Discussion}

\subsection{Some Alternative Methods for Constructing Bivariate Distribution}

Family. First proposal is as follows: We obtain by mixing $H_{1}$ in (7) and $H_{2}$ in (8) with respective mixing probabilities $\frac{1+\lambda}{2}$ and $\frac{1-\lambda}{2}$, then we have

$$
H_{4}(x, y)=\frac{(1-\lambda)}{2} F_{x} F_{y}+\frac{(1+\lambda)}{2} F_{x y}-\lambda F_{x y} \bar{F}_{x y},
$$

where $\lambda \in[-1,1]$. Note that, $H_{4} \in F\left(F_{x}, F_{y}\right)$. In fact, Dolati and Úbeda-Flores (2009) 8 proposed two different distribution families and made their conclusions on these two families. Therefore, $H_{4}$ will be similar to their proposed families.

Second proposal is as follows: Let $\left(X_{1}, Y_{1}\right)$ and $\left(X_{2}, Y_{2}\right)$ be two independent random vectors with different bivariate distributions $F_{x} F_{y}$ and $F_{x y}$. Then we consider the random pair $\left(V_{1}, V_{2}\right)$ as follows:

$$
\left(V_{1}, V_{2}\right)=\left\{\begin{array}{l}
\left(\min \left\{X_{1}, X_{2}\right\}, \min \left\{Y_{1}, Y_{2}\right\}\right), \text { with probability } \frac{1+\lambda}{2} \\
\left(\max \left\{X_{1}, X_{2}\right\}, \max \left\{Y_{1}, Y_{2}\right\}\right), \text { with probability } \frac{1-\lambda}{2},
\end{array}\right.
$$

where $-1 \leq \lambda \leq 1$. Then the distribution of $\left(V_{1}, V_{2}\right)$ is given by

$$
H_{5}(x, y)=(1+\lambda) F_{x} F_{y}+\frac{(1+\lambda)}{2}\left[F_{x} F_{y} \bar{F}_{x y}+F_{x y} \bar{F}_{x} \bar{F}_{y}\right]-\lambda F_{x y} F_{x} F_{y} .
$$


Here, marginal distributions respectively are $H_{5 x}=(1+\lambda) F_{x}-\lambda F_{x}^{2}$ and $H_{5 y}=$ $(1+\lambda) F_{y}-\lambda F_{y}^{2}$. Note that, $H_{5}$ does not belong to $\mathcal{F}\left(F_{x}, F_{y}\right)$ and its marginals are represented by univariate transmuted distribution as in $H_{3}$ given by the eq. (9).

The genesis of the third proposal is based on the QRTM technique of [20], and Rüschendorf's Method of [17]. Then we define a function on the unit square as

$$
g(u, v)=u v+k(u, v)
$$

where $k(u, v)=0$ at the endpoints of the unit square, with $\frac{-\partial^{2} k(u, v)}{\partial u \partial v} \leq 1$. Specially, by choosing $k(u, v)=\lambda u v(1-u)(1-v)$, with $u=F_{x}$ and $v=F_{y}$ the eq. (12) indicates well-known bivariate distribution which is called as Farlie-GumbelMorgenstern (FGM) distribution (see Farlie, 1960, 9] and Gumbel, 1960, [10]). Note also that, both $g(u, 1)$ and $g(1, v)$ are indicate the QRTM technique of [20]. In the light of these works, we consider

$$
k(u, v)=\lambda u v-\lambda C_{u v}^{2}+\lambda(1+\lambda) C_{u v} \bar{C}_{u v},
$$

where $C_{u v}$ is a bivariate copula, and $\bar{C}_{u v}$ is a survival copula. Accordingly, the third proposal is introduced as

$$
H_{6}(x, y)=(1+\lambda) F_{x} F_{y}-\lambda F_{x y}^{2}+\lambda(1+\lambda) F_{x y} \bar{F}_{x y},
$$

with the marginal distributions respectively are $(1+\lambda) F_{x}-\lambda F_{x}^{2}$ and $(1+\lambda) F_{y}-$ $\lambda F_{y}^{2}$. The eq. (13) can be seen as another mixture of the eq. (5) and eq. (6). In other words, for $\lambda=-1$, eq. (13) reduces to eq. (6), and for $\lambda=1$, the eq. (13) reduces to eq. (5). Note that, $H_{6}$ does not belong to $\mathcal{F}\left(F_{x}, F_{y}\right)$ and its marginals are represented by univariate transmuted distribution. Unlike $H_{3}$ and $H_{5}$ alternatives, $H_{6}$ contains the independence class. In other words, for $\lambda=0, H_{6}$ produces $F_{x} F_{y}$ which is the member of $\mathcal{F}\left((1+\lambda) F_{x}-\lambda F_{x}^{2},(1+\lambda) F_{y}-\lambda F_{y}^{2}\right)$.

Thus, we just propose $H_{4}, H_{5}$ and $H_{6}$ as alternative bivariate distributions. By the next section, considering the special choice of $F_{x y}$, we will make a comparison amongst to works of [ [8, [13], 6], [21] ], according to their Spearman's rank correlation coefficients.

3.2. Spearman's Rho Measures for the New Families of Bivariate Distributions. For $F \in \mathcal{F}\left(F_{x}, F_{y}\right)$, Spearman's rho can be expressed as

$$
\rho_{s}=12 \int_{R} \int_{R}\left\{F_{x y}-F_{x} F_{y}\right\} d F_{y} d F_{x}
$$

(see, [19]).

FGM distribution, a well-known bivariate distribution, will be considered, and calculations of Spearman's rho will be made according to this base distribution. The Farlie-Gumbel-Morgenstern (FGM) family of bivariate distributions are given by $F_{x y}=F_{x} F_{y}\left[1+\theta \bar{F}_{x} \bar{F}_{y}\right]$, for $\theta \in[-1,1]$. Note that, $\rho_{s}=\frac{\theta}{3}$ (see 9 and $[10]$ ). 
The calculated coefficient of Spearman's rho for $H_{4}$ (in eq. (10) ) can be obtained by

$$
\begin{aligned}
\rho_{s}^{H_{4}} & =\left(\frac{1+\lambda}{2}\right) \rho_{s}^{F}-12 \lambda \int_{R} \int_{R} F_{x y} \bar{F}_{x y} d F_{y} d F_{x} \\
& =\frac{1}{6} \theta-\frac{1}{75} \lambda \theta^{2}-\frac{1}{3} \lambda .
\end{aligned}
$$

In fact, this result overlaps with the result of 8 (see, end of Section 3). Because we created a mixture distribution by $H_{4}$ of their proposed distributions. According to their reported results, $\rho_{s}^{H_{4}}$ attains minimum value as $-\frac{77}{150} \cong-.513$ at $(\theta=$ $-1, \lambda=1)$ and has a maximum value as as $\frac{77}{150} \cong .513$ at $(\theta=1, \lambda=-1)$. by

The calculated coefficient of Spearman's rho for $H_{5}$ (in eq. (11)) can be obtained

$$
\rho_{s}^{H_{5}}=\frac{1}{3}+\frac{1}{12} \theta+\frac{11}{300} \lambda^{2} \theta-\frac{1}{3} \lambda^{2} .
$$

$\rho_{s}^{H_{5}}$ has a minimum value as -.12 at $(\theta=-1, \lambda= \pm 1)$ and has a maximum value as $\frac{5}{12} \cong .417$ at $(\theta=1, \lambda=0)$. by

The calculated coefficient of Spearman's rho for $H_{6}$ (in eq. $(13)$ ) can be obtained

$$
\rho_{s}^{H_{6}}=\frac{\theta \lambda^{2}\left(\lambda^{2}+2 \theta+35\right)}{150} .
$$

$\rho_{s}^{H_{6}}$ has a minimum value as $\frac{-34}{150} \cong-.227$ at $(\theta=-1, \lambda= \pm 1)$ and has a maximum value as $\frac{38}{150} \cong .253$ at $(\theta=1, \lambda= \pm 1)$.

For the comparison, we calculate coefficient of Spearman's rho for $H_{3}$ (in eq. (9)) proposed by 13 as follows:

$$
\rho_{s}^{H_{3}}=\frac{1}{3}+\frac{1}{6} \theta+\frac{1}{75} \theta^{2}+\frac{11}{150} \lambda^{2} \theta-\frac{1}{3} \lambda^{2} .
$$

$\rho_{s}^{H_{3}}$ has a minimum value as $\frac{-34}{150} \cong-.227$ at $(\theta=-1, \lambda= \pm 1)$ and has a maximum value as $\frac{77}{150} \cong .513$ at $(\theta=1, \lambda=0)$.

We can see that the lower bound of $\rho_{s}^{H_{3}}$ is the same of $\rho_{s}^{H_{6}}$, and the upper bound is the same as $\rho_{s}^{H_{4}}$. Furthermore, in 6 it was reported that for $\theta \in[-1,0]$, $\rho_{s} \in\left[-\frac{1}{4}, \frac{1}{3}\right]$ and in 21$]$ it was reported that for $\theta \in[-1,0], \rho_{s} \in\left[-\frac{1}{3}, \frac{1}{3}\right]$.

The upper bounds of Spearman rho for $H_{3}, H_{4}$ and $H_{5}$ yield a wider range than FGM does. According to the comparison for the lower bound, $H_{4}$ offers wider range than FGM does.

\section{Results}

We make an in-depth review of the work which is the first of the bivariate extensions of QRTM available in the literature. A discussion is made that this first 
extension is a bivariate distribution under which conditions. The result of Theorem 1 shows that this bivariate extension cannot be a bivariate distribution function in some values of transmutation parameter $\lambda$. Therefore, other extensions existing in literature derived from or similar to this extension proposal may also need to be explored in detail.

To succeed to extend the univariate QRTM to the bivariate case, three new bivariate distribution families have been proposed as an alternative to the bivariate distribution families obtained using bivariate order statistics. We have shown that $H_{4}$ has a wider range of dependence measure than FGM has when the base distribution is the specially selected bivariate FGM distribution family is taken. Furthermore, $H_{5}$ has also a successful range according to the positive dependence measure.

Author Contribution Statements The authors contributed equally and significantly in this manuscript, and they read and approved the final manuscript.

Declaration of Competing Interests The authors declare that they have no known competing financial interests or personal relationships that could have appeared to influence the work reported in this paper.

Acknowledgement We thank the two anonymous reviewers whose comments/ suggestions helped improve and clarify this manuscript.

\section{REFERENCES}

[1] Alizadeh, M., Merovci, F., Hamedani, G. G., Generalized transmuted family of distributions: properties and applications, Hacettepe Journal of Mathematics and Statistics, 46 (2017), 645-667.

[2] Asadian, N., Amini, M., Bozorgnia, A., Some concepts of negative dependence for bivariate distributions with applications, Journal of Mathematical Extension, 4 (1) (2009), 43-59.

[3] Baker, R., An order-statistics-based method for constructing multivariate distributions with fixed marginals, Journal of Multivariate Analysis, 99 (10) (2008), 2312-2327, https://dx.doi.org/10.1016/j.jmva.2008.02.019.

[4] Bakouch, H. S., Jamal, F., Chesneau, C., Nasir, A., A new transmuted family of distributions: Properties and estimation with applications, working paper or preprint, Sept. 2017.

[5] Barlow, R. E., Proschan, F., Statistical theory of reliability and life testing: probability models, Tech. rep., Florida State Univ Tallahassee, 1975, https://dx.doi.org/10.2307/1268641.

[6] Bekçi, M., Yılmaz, M., Construction of bivariate distribution by mixing positively dependent and negatively dependent distributions, International Journal of Statistics and Applications, 9 (4) (2015), 122-1127, https://dx.doi.org/10.5923/j.statistics.20190904.04.

[7] Bourguignon, M., Ghosh, I., Cordeiro, G. M., General results for the transmuted family of distributions and new models, Journal of Probability and Statistics, 2016 (2016), https://dx.doi.org/10.1155/2016/7208425.

[8] Dolati, A., Ubeda-Flores, M., Constructing copulas by means of pairs of order statistics, Kybernetika, 45 (6) (2009), 992-1002.

[9] Farlie, D. J. G., The performance of some correlation coefficients for a general bivariate distribution, Biometrika, 47 (3/4) (1960), 307-323, https://dx.doi.org/10.2307/2333302. 
[10] Gumbel, E. J., Bivariate exponential distributions, Journal of the American Statistical Association, 55 (292) (1960), 698-707, https://dx.doi.org/10.1080/01621459.1960.10483368.

[11] Merovci, F., Alizadeh, M., Hamedani, G. G., Another generalized transmuted family of distributions:properties and applications, Austrian Journal of Statistics, 45 (3) (Jun. 2016), 71-93, https://dx.doi.org/10.17713/ajs.v45i3.109.

[12] Merovci, F., Alizadeh, M., Yousof, H. M., Hamedani, G. G., The exponentiated transmuted-g family of distributions: theory and applications, Communications in Statistics - Theory and Methods, 46 (21) (2017), 10800-10822, https://dx.doi.org/10.1080/03610926.2016.1248782.

[13] Mirhoseini, S., Dolati, A., Amini, M., On a class of distributions generated by stochastic mixture of the extreme order statistics of a sample of size two, arXiv preprint arXiv:1904.04287 (2019).

[14] Mirhosseini, S. M., Amini, M., Dolati, A., On a general structure of the bivariate fgm type distributions, Applications of Mathematics, 60 (1) (2015), 91-108, https://dx.doi.org/10.1007/s10492-015-0086-6.

[15] Oluyede, B. O., On local dependence and stochastic inequalities with applications to contingency tables, Applied mathematics and computation, 151 (3) (2004), 801-813, https://dx.doi.org/10.1016/s0096-3003(03)00537-x.

[16] Rezaei, S., Marvasty, A. K., Nadarajah, S., Alizadeh, M., A new exponentiated class of distributions: Properties and applications, Communications in Statistics - Theory and Methods, 46 (12) (2017), 6054-6073, https://dx.doi.org/10.1080/03610926.2015.1116579.

[17] Rüschendorf, L., Construction of multivariate distributions with given marginals, Annals of the Institute of Statistical Mathematics, 37 (2) (1985), 225-233, https://dx.doi.org/10.1007/bf02481093.

[18] Sarabia, J. M., Raja, A. V., Asha, G., Bivariate distributions with transmuted conditionals: models and applications, Communications in Statistics - Theory and Methods, 49 (1) (2020), 221-242, https://dx.doi.org/10.1080/03610926.2018.1536785.

[19] Schweizer, B., Wolff, E. F., On nonparametric measures of dependence for random variables, The Annals of Statistics, 9 (4) (1981), 879 - 885, https://dx.doi.org/10.1214/aos/1176345528.

[20] Shaw, W. T., Buckley, I. R. C., The alchemy of probability distributions: beyond GramCharlier expansions, and a skew-kurtotic-normal distribution from a rank transmutation map, ArXiv e-prints (Jan. 2009).

[21] Ünözkan, H., Yllmaz, M., Construction of continuous bivariate distribution by transmuting dependent distribution, Cumhuriyet Science Journal, 40 (4) (2019), 860-866, https://dx.doi.org/10.17776/csj.618236. 